\title{
ESTUDIOS SOCIO-ANTROPOLÓGICOS CON NIÑOS Y NIÑAS TRABAJADORES. UNA APUESTA REFLEXIVA SOBRE DOS EXPERIENCIAS EN AMÉRICA LATINA: MÉXICO Y ARGENTINA ${ }^{1}$
}

\author{
$\mathrm{M}^{\mathrm{a}}$ Eugenia RAUSKY ${ }^{2}$. \\ Begoña LEYRA FATOU ${ }^{3}$
}

\begin{abstract}
RESUMEN
Este artículo se inscribe en el campo de los estudios sociales sobre infancia y tiene como objetivo desarrollar una contribución reflexiva sobre dos experiencias de investigación conducidas con niños y niñas trabajadores en dos contextos urbanos de Latinoamérica diferentes, uno en México y otro en Argentina.

PALABRAS CLAVE: REFLEXIVIDAD, TRABAJO INFANTIL, METODOLOGÍAS DE INVESTIGACIÓN, NIÑOS Y NIÑAS, LATINOAMÉRICA.
\end{abstract}

\begin{abstract}
This paper is part of the field of social studies of childhood and aims to develop a thoughtful contribution on two research conducted with working children in two urban contexts of different Latin American countries: Mexico and Argentina

KEY WORDS: REFLEXIVITY, CHILD LABOUR, METODOLOGY OF RESEARCH, CHILDREN, LATINOAMERICA
\end{abstract}

\section{RÉSUMÉ}

Cet article fait partie du domaine des études sociales de l'enfance et vise à développer une contribution réfléchie sur deux expériences menées recherche avec des enfants, qui travaillent dans deux contextes urbains différents en Amérique latine: un au Mexique et un en Argentine.

\footnotetext{
${ }^{1}$ Una primera versión de este trabajo se presentó en el $8^{\circ}$ Congreso Internacional CEISAL (Salamanca, julio de 2016).

${ }^{2}$ Investigadora del CIMeCS, Unidad de investigación del IdIHCS-UNLP/CONICET- de la Facultad de Humanidades y Cs. de la Educación de la Universidad Nacional de La Plata (Argentina). Calle: 51 e/ 124 y 125. Edificio C. Oficina 320. CP. 1925. eugeniarausky@gmail.com

3. Investigadora del Departamento de Trabajo Social y Servicios Sociales-Facultad de Trabajo Social y Vice-directora del Instituto Complutense de Estudios Internacionales (ICEI) de la Universidad Complutense de Madrid (España). Campus de Somosaguas. 28223, Pozuelo de Alarcón, Madrid. mbleyra@ucm.es
}

Fecha de recepción del artículo: Agosto 2016

Fecha de evaluación: Marzo 2017 
MOTS-CLEFS :REFLEXIVITE, -TRAVAIL DES ENFANTS-METHODOLOGIES DE RECHERCHE - ENFANTS- AMERIQUE LATINE.

\section{Introducción}

En el campo de las ciencias sociales la invocación a la reflexividad o a las prácticas de investigación reflexivas ha ganado terreno en el contexto de indagaciones etnográficas y cualitativas. Prácticamente ya nadie pone en cuestión la centralidad que adquiere para el proceso de conocimiento la revisión permanente sobre los supuestos y sobre las aproximaciones metodológicas que nos guían. Se supone que esto nos vuelve mejores investigadores/as y que esta práctica deviene consecuentemente en investigaciones de mayor calidad.

De las distintas concepciones sobre la reflexividad ${ }^{4}$, la consideramos aquí como instancia capaz de expresar la conciencia del investigador, hecho que redunda en la posibilidad de mejorar los instrumentos de investigación científica, su capacidad observacional, representativa e interpretativa. La reflexividad supone en definitiva "pensar lo que se hace" (Hidalgo, 2006).

En esta comunicación buscamos compartir un ejercicio reflexivo basándonos en dos experiencias de investigación socio-antropológicas desarrolladas en distintos contextos nacionales latinoamericanos (México y Argentina), que se han interrogado por un mismo fenómeno, el de los niños y niñas que trabajan en ámbitos urbanos.

Si bien el proceso reflexivo atraviesa las diferentes instancias de un estudio: desde las preguntas iniciales al trabajo de campo, del análisis de los datos a la escritura, aquí nos concentraremos en el proceso de construcción del objeto de conocimiento y en las decisiones relativas al trabajo de campo: ¿Qué caminos hemos recorrido en la construcción de este objeto/sujeto de investigación? ¿Por qué escogimos a los niños/as que trabajan? ¿Qué perspectivas metodológico-técnicas empleamos? ¿Cómo recortamos las unidades de análisis? ¿Qué tensiones o dificultades se presentaron en el trabajo de campo? ¿Cómo se encauzaron los dilemas éticos -por cierto, muy presentes en este campo de estudios-? Estas son algunas de las preguntas que aquí abordaremos buscando fundamentalmente hacer una contribución metodológica reflexiva a los estudios sobre niñez. Proponemos entonces un ejercicio crítico sobre las condiciones de producción del conocimiento en nuestras investigaciones empíricas en las que se han encontrado varios elementos en común como así también algunas divergencias.

\section{Sobre las investigaciones: construcción del objeto, recorte espacio temporal, y preguntas de investigación}

Las investigaciones de las que aquí vamos a dar cuenta comparten prácticamente un mismo trayecto temporal ya que han comenzado a principios del cambio de siglo, durante los primeros años del siglo XXI. En dicho momento el contexto latinoamericano -más allá de las especificidades de cada uno de los países en que se emplazaron- compartía un escenario común: la creciente pobreza, exclusión y vulnerabilidad cobraban cada vez más centralidad en Latinoamérica producto de las políticas neoliberales de los años 90, haciendo de sus consecuencias algo devastador para sus habitantes, poniendo en jaque la integración social e hipotecando el futuro de sucesivas generaciones.

Remarcar esto es de fundamental importancia, ya que el trabajo infantil suele llevarse adelante en contextos de pobreza y creciente vulnerabilidad, algo que se encargaron de destacar numerosísimos estudios. Ahora bien, si la interrelación pobreza/inserciones laborales tempranas

\footnotetext{
${ }^{4}$ Cabe destacar que la noción de reflexividad contiene múltiples acepciones. Tal como señala Hidalgo (2006) a grandes rasgos, pueden reconocerse dos ejes en torno a los cuales se puede considerar: uno, que la define como instancia para mejorar los instrumentos de investigación científica, su capacidad observacional, representativa e interpretativa; o como una instancia para interrogarse sobre la propia práctica de investigación. Otro, en función del modo en que se encuentra involucrada la subjetividad del investigador: del narcisimo al objetivismo, pasando por posiciones intermedias.
} 


\section{Papeles de Trabajo No 33 - Julio 2017 - ISSN 1852-4508 \\ Centro de Estudios Interdisciplinarios en Etnolingüística y Antropología Socio-Cultural}

resultaba insoslayable en los estudios sobre este campo temático, es necesario reconocer que no es únicamente la infancia pobre la que trabaja, ya que son conocidas, aunque escasamente problematizadas en el campo de las ciencias sociales, las manifestaciones del trabajo durante la niñez en sectores medios o medio-altos de la sociedad. Casos como el de las carreras artísticas (cine, teatro o televisión), deportivas, o las incursiones en el mundo de la publicidad son expresiones elocuentes sobre ello. Así surge un primer interrogante ¿por qué estudiar a la niñez pobre? ¿Por qué no considerar las distintas expresiones del trabajo infantil haciendo un análisis transversal que contemplara distintos sectores sociales?

Justificar el recorte de una investigación lleva a pensar en razones de distinta naturaleza -teóricas y prácticas- que terminaron por conducirnos hacia el estudio de la niñez trabajadora perteneciente a sectores extremadamente pobres. En buena medida creemos que esa elección estuvo condicionada tanto por nuestra incorporación previa a equipos de investigación vinculados con estudios sobre sectores pobres urbanos, como porque sentimos una particular sensibilidad hacia los niños y niñas, quienes permanecían invisibilizados en este campo de estudios: mucho se conocía sobre procesos de crianza, alimentación, nutrición y educación en la infancia, pero poco sobre el trabajo infantil y menos aún desde una perspectiva que integrara las voces de los niños y niñas. Sin conocernos previamente, ambas investigadoras fuimos construyendo un recorrido con muchos puntos en común para el abordaje del trabajo infantil urbano, que aquí proponemos socializar.

La investigación realizada en Argentina fue desarrollada en un barrio con características de pobreza estructural situado en la periferia de la ciudad de La Plata -capital de la provincia de Buenos Aires-. A medida que fuimos conociendo el lugar -a raíz de la participación en un proyecto de extensión universitaria- y fuimos detectando las diferentes problemáticas, encontramos que en varios de los hogares había niños y niñas que desarrollaban actividades laborales. Lo más frecuente era verlos junto a algunos miembros de su familia arriba de los carros (yendo o viniendo de la recorrida por las calles de la ciudad) o trabajando en sus viviendas en la descarga y clasificación del material recolectado en las calles.

Teniendo en cuenta las posibilidades de acceso que se abrieron y el conocimiento de las características del lugar decidimos llevar a cabo la investigación en dicho barrio. Complementando luego las observaciones en los espacios de trabajo en que estos niños/as hacían sus actividades: la calle. Tal como plantea Guber (1991), la accesibilidad al escenario y a las personas del lugar se constituyeron en un criterio clave en la elección/construcción de la unidad espacial y la unidad de análisis.

El recorte analítico lo fuimos construyendo y perfeccionando a medida que comenzamos a visualizar un conjunto de particularidades en nuestros primeros acercamientos empíricos, al tomar contacto con el barrio. Por un lado, encontramos que el trabajo de los niños y niñas se desarrollaba en el marco de emprendimientos familiares: los niños llevaban a cabo actividades laborales junto a sus padres/madres y/o algún familiar cercano. Por otro lado, dichas actividades que consistían en el cirujeo ${ }^{5}$, la mendicidad o la venta de productos en algunas de las esquinas de la ciudad, se desplegaban en el espacio público, o sea, en la calle. Así comenzamos a delinear el proceso de construcción del objeto de investigación en torno al cual surgieron una serie de interrogantes: ¿qué características asume la relación trabajo-familia? ¿En qué medida los vínculos sociales que se expresan al interior de las familias ayudan a comprender la emergencia de situaciones de trabajo infantil? ¿Cómo operan en este proceso las valoraciones acerca de los roles de cada miembro del hogar en la división de tareas? ¿Qué lugar ocupan allí los niños/as? ¿Qué sentidos cobra el trabajo infantil? ¿Cómo se construye la niñez? ¿Qué especificidad adquiere el fenómeno laboral al ser desarrollado en las calles? ¿De qué modos los niños/as y sus familiares practican y representan el espacio de trabajo?

En el conjunto de estos interrogantes había dos grandes núcleos de cuestiones que sabíamos tenían especial relevancia en el análisis del fenómeno: uno vinculado al estudio del ámbito familiar, otro

\footnotetext{
${ }^{5}$ En Argentina cirujeo significa buscar o escoger desperdicios en basureros.
} 


\section{Papeles de Trabajo No 33 - Julio 2017 - ISSN 1852-4508 \\ Centro de Estudios Interdisciplinarios en Etnolingüística y Antropología Socio-Cultural}

relacionado con la exploración de los lugares de trabajo. La incursión analítica por estos dos ámbitos nos daría las pistas adecuadas para vislumbrar las experiencias asociadas al trabajo infantil, y con ello podríamos comenzar a develar algunas de las lógicas subyacentes en la comprensión de la aparición de situaciones de este tipo.

La particularidad del abordaje que planteamos radicó en un interés por conocer este fenómeno considerando varios planos que interactuaran de manera simultánea, evitando centrarnos solo en un aspecto, sino articulando un conjunto de elementos que intervienen en la comprensión del mismo. Esto trajo aparejada una mirada que recuperara tres ejes: a) las vivencias del niño/a y actores cercanos en relación al trabajo, o sea, cómo el niño trabajador y allegados experimentan el trabajo infantil. Además de considerar las características objetivas del trabajo (tipo de inserción, ingresos, etc.), nos preguntamos qué significa para el niño/a trabajador y su familia realizar esa actividad con todas las connotaciones que ello tenga, confrontando estas miradas con las del sentido común; b) la visualización y análisis del trabajo infantil en el conjunto de las estrategias de reproducción de las familias, esto equivale a indagar el papel que ocupa esta práctica; cómo se integra al resto de las prácticas tendientes a la reproducción, lo que conlleva necesariamente una mirada hacia la unidad doméstica situándonos en la esfera de las relaciones sociales entre sus miembros, viendo cómo se vinculan, qué criterios guían la división del familiar del trabajo, cómo se decide quién trabaja y quién no, cómo operan las diferencias de género y de generaciones en la organización doméstica, entre otras cosas y; c) la recuperación de la espacialidad del trabajo, o sea, la visualización de los espacios en que desarrollan sus trabajos y las vivencias asociadas a los mismos.

En el caso de México como hipótesis de partida, se planteó demostrar que la realización de estas actividades laborales tiene una incidencia diferenciada en el desarrollo y socialización en las niñas trabajadoras respecto a los niños y que la lectura y valoración que en el ámbito familiar y comunitario se hace de estos trabajos también es diferente, generando desde la infancia las desigualdades de género. Con esta investigación se buscó visibilizar esta práctica laboral y analizar la repercusión que tiene en el desarrollo integral de las niñas, así como también describir los aspectos socioculturales que la rodean, tratando de considerar las diferentes justificaciones que sobre el trabajo de las niñas se construyen, como algo coyuntural, educativo, cultural o como una estrategia debido a la falta de recursos entre sus familias. Así mismo se reflexionó sobre la lectura y valoración que de estas actividades se hacen, por parte de las propias niñas, de sus familias y unidades domésticas y de otros agentes sociales (de las comunidades, organizaciones no gubernamentales y de la sociedad civil de su entorno).

La búsqueda de esta investigación se orientó entonces a identificar, visibilizar y analizar las diferentes formas de trabajo que realizan las niñas en un contexto determinado, la Ciudad de México, analizando las condiciones de trabajo y las diferentes interpretaciones y lecturas que se hacen de estas teniendo en cuenta la visión y conceptualización cultural del fenómeno; analizando estas actividades como parte de la configuración de identidad de género y como parte del proceso socializador de las niñas enfatizando en los mecanismos a través de los cuales en la familia y en el trabajo se crean las diferencias y las desigualdades de género.

Partiendo de premisas de la antropología del trabajo, del género, la economía y el parentesco se pretendía estudiar las causas que fortalecen y perpetúan esta consideración "silenciada" por una gran parte de la sociedad e infravalorada por otra. Las preguntas buscaron elucidar ¿cuál era la valoración del trabajo infantil dentro y fuera del ámbito familiar y doméstico desde una perspectiva de género? ¿Cómo habían sido las trayectorias laborales familiares y cómo estas incidían en la configuración laboral de las niñas? ¿Cómo se "conciliaba" el trabajo y la escuela? ¿Qué procesos subyacían a las diferentes asignaciones de roles "tradicionalmente femeninos" (que se desvelan en los trabajos realizados por las niñas) o "tradicionalmente masculinos"?

Si bien muchas de las preguntas que subyacieron a ambas investigaciones fueron similares, había un elemento marcadamente distinto en ambas: la necesidad de orientar la mirada desde una perspectiva de género estaba presente en una investigación (México) y no en la otra (Argentina), que si bien se interrogaba por los modos distintos en que el trabajo infantil incidía en niños y niñas, no centraba la mirada desde el enfoque de género. Entendemos que en esta definición 


\section{Papeles de Trabajo No 33 - Julio 2017 - ISSN 1852-4508 \\ Centro de Estudios Interdisciplinarios en Etnolingüística y Antropología Socio-Cultural}

teórica intercedieron dos elementos. Por un lado, las especificidades de cada contexto: en la sociedad mexicana los estereotipos tradicionales de género están profundamente arraigados, al menos mucho más que en la sociedad Argentina. Por otro lado, la formación específica y el interés teórico de una de las investigadoras en la perspectiva de género que conllevó una observación del fenómeno atendiendo a esa clave de lectura.

\section{Aportes de los childhood studies para estudiar el trabajo infantil: el lugar de los niños y niñas en la investigación social y las decisiones sobre la producción de los datos}

La formulación de los interrogantes supuso la toma de decisiones respecto de la perspectiva teórica y metodológica que utilizaríamos. Si bien los enfoques socio-antropológicos sobre pobreza, estrategias de reproducción, economía y trabajo fueron claves en nuestras indagaciones empíricas, la mirada general sobre el fenómeno a abordar la proveyeron los childhood studies ${ }^{6}$. De los diferentes aportes que desde este campo se realizaron para estudiar a la infancia, interesa subrayar aquí uno en particular: la asunción de que los niños y niñas no son meros agentes que internalizan pasivamente la cultura adulta, sino que ellos son parte de la misma y contribuyen a su reproducción y cambio (Eder y Corsaro, 1999).

Dicha premisa trajo aparejada un replanteamiento a nivel metodológico vinculado al lugar que ocupan los niños y niñas en la investigación social. Fundamentalmente, los precursores de esta perspectiva sostienen y reclaman el derecho a que la voz de niños y niñas sea tomada en cuenta seriamente en las investigaciones.

Quienes se nuclean en tales perspectivas comienzan a plantear que si se prescinde de la infancia en la investigación social se observa solamente una parte del conjunto, en cambio, si se la reconoce, es posible analizar las relaciones de poder entre los adultos y los niños y niñas, la distribución generacional de roles, la atribución diferenciada de recursos sociales y los conflictos de intereses que se producen como consecuencia de esto (Gaitán, 2006). A diferencia de los trabajos que antecedieron a la nueva corriente de estudios sobre infancia, que sobretodo en el campo de la sociología centraron su interés en los procesos de socialización y en el análisis del comportamiento de las instituciones encargadas de llevar adelante tal proceso -la familia y la escuela-, y que por ende, no tomaban a la infancia en sí como objeto de estudio, sino su socialización (entendida como proceso unidireccional desde el adulto hacia el niño) y las desviaciones que se derivaban de este proceso; esta corriente enfatiza la necesidad de comprender a la infancia y los procesos en los que se inscribe atendiendo a su punto de vista.

Así como en los últimos años a nivel teórico los nuevos estudios sociales de la infancia ayudaron a repensar y cuestionar los modos de concebir la niñez y su lugar en la sociedad, tales planteamientos tuvieron su correlato en el plano metodológico, originando una serie de aportes respecto del modo de hacer investigación social empírica con este grupo de edad (Rausky, 2010). Enfatizando la importancia de tomar en cuenta la voz de los niños y niñas (Christensen, 2004) los investigadores del campo han consensuado que en relación a los abordajes metodológicos las aproximaciones de tipo cualitativas y/o etnográficas (la mayoría de los trabajos responden a este enfoque) son las que más pueden acercarse a tales objetivos, poniendo énfasis en la idea de participación de los niños y niñas.

El acento puesto en la implementación de los métodos y técnicas participativas se sustenta en las ventajas que presentan. A saber: a) prometen el acceso directo a la perspectiva de los niños y niñas y, b) en tanto respetan la capacidad de acción de ellos, son emancipadoras y democráticas. Como argumenta Grover (2004) al permitir la participación activa de los niños y niñas en el proceso de investigación, se realza su estatus como individuos con derechos inherentes a la participación en la sociedad y a ser escuchados. En la medida en que desde las ciencias sociales se reconceptualiza el lugar de la infancia en el mundo y se busca dar cuenta de su capacidad de

\footnotetext{
${ }^{6}$ Sirota (2001) proporciona un muy buen análisis al respecto de cómo se ha constituido y evolucionado el campo. Si bien enfatiza sobre lo ocurrido en el mundo francoparlante, también incorpora elementos sobre las producciones angloparlantes. Gaitán (2006) también hace una sistematización completa sobre la evolución y estado actual del campo.
} 
acción, se reconoce que su perspectiva es fundamental para alcanzar tales fines, pero ¿cómo alcanzarla? Por medio de la participación.

Mientras que en general las ciencias sociales hasta ese momento no consideraban a la infancia para hacer investigaciones que se refieran a su vida, intereses o sus problemas, estas aproximaciones teóricas defienden lo contrario (Pollock, 1983; Alarcón, 1994; Bar-Din, 1995; Boyden y Ennew, 1997; Casas, 1998; Castillo, 2004; Rodríguez, 2005; Gaitán, 2006; Lahire, 2007), y esto implica que niños y niñas tienen capacidad para identificar sus problemas e indagar sobre la naturaleza y alcance de los mismos.

Gaitán y Liebel (2011) sugieren que hay tres posibles modelos en la investigación de la infancia, que son los siguientes:

Tabla 1. Modelos de investigación de la infancia

\begin{tabular}{|c|c|c|}
\hline $\begin{array}{l}\text { Investigación sobre niños } \\
\text { y niñas }\end{array}$ & Niños y niñas como objetos & \begin{tabular}{|lll}
$\begin{array}{l}\text { Perspectiva } \\
\text { adultas }\end{array}$ & de & personas \\
\end{tabular} \\
\hline $\begin{array}{l}\text { Investigación con niños y } \\
\text { niñas }\end{array}$ & $\begin{array}{llll}\text { Niños y niñas como } \\
\text { informantes } \\
\begin{array}{l}\text { Niños y niñas como co- } \\
\text { investigadoras }\end{array}\end{array}$ & $\begin{array}{l}\text { Perspectiva de personas } \\
\text { adultas } \\
\text { Perspectivas de niños y niñas y } \\
\text { personas adultas }\end{array}$ \\
\hline $\begin{array}{l}\text { Investigación } \\
\text { encabezada por niños y } \\
\text { niñas }\end{array}$ & $\begin{array}{l}\text { Personas adultas como co- } \\
\text { investigadoras } \\
\text { Personas adultas como asesoras }\end{array}$ & $\begin{array}{l}\text { Perspectiva de niños y niñas } \\
\text { Perspectiva de niños y niñas }\end{array}$ \\
\hline
\end{tabular}

Fuente: Gaitán y Liebel (2011: 173)

Casi resulta obvio decir que desde un punto de vista centrado en los niños y las niñas, las diferentes combinaciones que pueden darse dentro de los dos últimos modelos son las que resultan más deseables. La participación activa de niños y niñas en la investigación de la infancia es considerada importante, porque ellos son los que mejor pueden representar o transmitir su posición.

En concordancia con esta mirada, nuestras investigaciones pusieron en el centro la necesidad de acudir a la perspectiva infantil a través de una aproximación metodológica flexible. Ahora bien, si esta perspectiva elegida ponía en el centro tal necesidad, en nuestra definición teníamos claridad al respecto de la inclusión de las voces adultas - madres, padres, hermanos mayores, docentes, referentes de la comunidad, etc.-, de modo que pudiésemos anclar el fenómeno relacionalmente y tener así un mapa más completo y complejo de los factores que confluían para entenderlo. Considerando que los niños y niñas son productores de sentidos, se entiende que pueden elaborarlos y presentarlos de modo variado (Cohn, 2007). Así, para acceder a la perspectiva de los niños y niñas empleamos entrevistas, observación participante y acudimos -en el caso de la investigación mexicana- a la producción de dibujos. La perspectiva infantil preferimos reservarla para la instancia de producción de la información y no en etapas previas o posteriores de la investigación.

Nos parece importante destacar que las entrevistas con niños/as asumieron ciertas características particulares. Se trató generalmente de encuentros un poco más breves que las desarrolladas con personas adultas, en las que predominó la lógica de la pregunta-respuesta. Preparamos un guion tentativo y orientador sobre los aspectos a conversar, al que se le fueron agregando ejes y dimensiones conforme avanzaba el trabajo de campo y los entrevistados-adultos y niños/asreferían aspectos importantes no anticipados por la investigadora.

En todo el camino recorrido asumimos como propio el planteo de Szulc (2004: 8)

\footnotetext{
"no negamos la incidencia de la edad de los sujetos sobre sus prácticas y representaciones, sino de tener en cuenta que la edad no es solo un hecho "biológico" sino también un estatus social e históricamente construido. Desnaturalizar la concepción cosificada y esencialista sobre la niñez nos conduce a reconocer que-aunque condicionados como
} 


\section{Papeles de Trabajo No 33 - Julio 2017 - ISSN 1852-4508 \\ Centro de Estudios Interdisciplinarios en Etnolingüística y Antropología Socio-Cultural}

todos por su edad- también los niños son sujetos activos y posicionados. El hecho de ser niños, condiciona su realidad cotidiana y sus interpretaciones de la misma, pero ello no los descalifica como actores reflexivos"

$\mathrm{Al}$ establecer los contactos con los niños y niñas - previa medicación y permiso de los adultos responsables-, tratamos de explicarles siempre quiénes éramos y qué hacíamos, de modo que se alejaran las dudas o temores que pudieran tener: "nuestro trabajo es aprender y conocer el trabajo que ustedes hacen, cómo es su vida, etc....". Creemos que en algunos casos esto fue correctamente comprendido, y en otros percibimos que no se alcanzaba a dimensionar nuestra tarea, probablemente por ser algo poco familiar en el mundo de quienes estudiamos, ya que esto mismo sucedió también con algunas de las personas adultas.

Los modos en que se nos identificó fueron varios: "las señoritas", "las asistentes sociales", "las chicas de la facultad". En el caso de la investigación realizada en la Argentina, como más arriba se comentó, el trabajo de campo se inició en un barrio ya conocido, en el que la investigadora llevaba adelante tareas de extensión universitaria, así la familiarización con el lugar y su gente hicieron que el acceso fuese mucho más sencillo de lo esperado. No obstante ello, en algunas oportunidades se presentaron dudas de los padres y de los propios niños frente a la investigación, las que se manifestaron en ocasiones durante las entrevistas: "pero...¿¿y esto para qué es?" o en la presencia física de alguno de los padres durante algunos minutos en los momentos en que manteníamos las conversaciones a solas con los niños/as.

Sin embargo, en la investigación de México otras observaciones sobre este proceso merecen ser explicitadas: una mujer, antropóloga, extranjera, adentrándose en los mercados de los "barrios bravos" de la ciudad, en las zonas "peligrosas" o "marginales", en el basurero y en espacios con gran índice de violencia podía resultar "poco recomendable". En México muchos llamaban a la investigadora la güera ${ }^{7}$ y ese hecho hacía que no pudiera pasar "desapercibida" allá donde fuera, lo que en ocasiones supuso un problema y en muchas otras fue una puerta que facilitó el acceso a espacios donde en principio ni imaginó que pudiera llegar. A pesar de que inicialmente no se conocían los códigos lingüísticos ni los códigos internos de los lugares de observación y a sabiendas de que el esfuerzo sería grande, merecía la pena el intento: desvelar la situación de las niñas trabajadoras, hacer énfasis en las diferencias, proyectar una mirada de género sobre esta realidad social, visible a todas luces, evidente y que no siempre es considerada, ni siquiera reconocida, ni mencionada en los muchos estudios y aportaciones que se hacen al fenómeno del trabajo infantil. El trabajo de campo exigía mucha concentración y dedicación, la etnografía con niños y niñas aún en antropología era desconocida, difícil de realizar, el acceso a la realidad de las niñas debía ser progresivo, cuidadoso y constante hasta ganar la confianza de la gente, de las familias trabajadoras, eliminar el halo que rodeaba en ocasiones de "extranjera robaniños". A lo largo de las entrevistas se tornó necesario aprender los códigos lingüísticos, las formas de expresión para no confundir, aprender a "hablar" el mexicano, una tarea más a las muchas que se acumulaban y todo eso generó, especialmente al inicio, sensaciones de cansancio, impotencia y soledad.

En relación con las dificultades ligadas a la producción de la información también compartimos y experimentamos una "particular" visión que tienen las niñas y niños de la propia realidad, especialmente con los conceptos abstractos de tiempo o espacio, la confusión en fechas de cumpleaños, edades o tiempo que llevan trabajando. Así mismo, en la investigación mexicana otro dato interesante resultó de la construcción del orden familiar, la cual variaba bastante, ya que destacaban aquellos miembros de la unidad doméstica importantes, omitiendo otros personajes (presencia o ausencia de padres, hermanos, hermanas que en realidad ya no estaban o a la inversa), dichas dificultades pudieron, en casi todos los casos, ser trabajadas a través del cruce de información con otros miembros de la unidad doméstica o de la propia observación, y en general, lo que sí constituyó un elemento básico en las entrevistas fue la frescura y naturalidad de la

\footnotetext{
${ }^{7}$ Persona de piel clara.
} 


\section{Papeles de Trabajo No 33 - Julio 2017 - ISSN 1852-4508 \\ Centro de Estudios Interdisciplinarios en Etnolingüística y Antropología Socio-Cultural}

información (con canciones, preguntas a las entrevistadoras y curiosidades entrañables), añadiendo datos interesantísimos que dentro de la "visión" adulta nunca hubieran sido revelados.

\section{¿Dónde? y ¿A quiénes?: las definiciones sobre los ámbitos de observación y las unidades de análisis}

¿Dónde contactar a los niños y niñas? ¿En los ámbitos en que desarrollan su trabajo? ¿En la escuela -en los casos en que asistían-? ¿En espacios públicos de sus barrios? ¿En sus hogares? Frente a las diferentes posibilidades que se abrieron en relación al ámbito físico o unidad espacial de la investigación, en ambos casos optamos por no reducir la mirada hacia un único lugar sino que nos vimos en la necesidad de explorar diferentes escenarios, en especial los ámbitos de trabajo y los barrios en que habitaban los niños y niñas. Así, otra de las convergencias en ambos estudios remitió a la necesidad de observar y seguir a esos niños y niñas en múltiples espacios.

Lógicamente el recorte también debía pensarse en función de la vida urbana de cada uno de los territorios, la Ciudad de México es capital de ese país y una de las más pobladas de Latinoamérica, mientras que la ciudad de La Plata es la capital de la provincia de Buenos Aires y cuenta con una población muy inferior a la de Ciudad de México. Al mismo tiempo, las tipologías de trabajo infantil urbano reconocidas en uno y otro lugar eran diferentes: mientras que en Argentina la modalidad familiar de trabajo infantil tenía una presencia cuantitativamente importante, en México no lo era tanto.

En la investigación Argentina priorizamos dos ámbitos: el barrio/sus hogares y el espacio de trabajo. Como la mayor parte de los niños y niñas llevaba a cabo sus actividades laborales en el espacio público, ya sea en carros que recolectaban basura, vendiendo flores en esquinas de la ciudad o mendigando, nos desplazamos hacia esos ámbitos -recortamos algunos espacios emblemáticos de la ciudad- y compartimos allí tiempo con ellos. A la vez durante algunos días acompañamos a una familia de cartoneros en la realización del trabajo de recolección.

En la investigación en México se debía precisar una zona que fuera abarcable físicamente y que tuviera una importante densidad comercial. Por este motivo se trabajó en los barrios y se seleccionaron siete espacios: el Mercado de La Merced y Tepito, que existían ya en tiempos prehispánicos y son paradigma de "zonas comerciales" en la ciudad, el Mercado de la colonia Morelos (considerado como el corazón de Tepito), el Mercado de Sonora, como uno de los más peculiares y típicos de la ciudad, y que debido a su cercanía con La Merced, ya forma parte de él, la Central de Abasto, que a pesar de encontrarse en otra delegación política, tiene muchas relaciones históricas con La Merced (que hasta 1982 fue el Mercado de Abasto de la ciudad) y hoy en día es uno de los mercados más grandes de América Latina, con una gran densidad de niños, niñas y adolescentes trabajadores dentro de sus grandísimas instalaciones, la colonia Santa María la Ribera, por encontrar allí a niñas trabajando en otros ámbitos de interés (tales como la maquila doméstica o las niñas cartoneras y por estar ahí situada una organización que trabajaba exclusivamente con niñas en situación de calle) y el basurero de Chimalhuacán que a pesar de salir de las fronteras del Distrito Federal, fue seleccionado por constituir uno de los lugres donde se encuentran niñas trabajando en la recogida y selección de basura.

Como ya argumentamos, en la medida en que en un caso la investigación se desarrollaría en un lugar conocido y familiar para la investigadora y en el otro no, la accesibilidad al campo en este último caso requirió de otro conjunto de estrategias. En México, para acceder inicialmente a los mercados, colonias, barrios y espacios seleccionados, especialmente en la primera fase, se acudió a organizaciones que trabajaban en las zonas de interés con el sector de infancia que se buscaba, accediendo a ellas tras un arduo repaso de directorios de organizaciones y asociaciones de infancia mexicanas. En general, es destacable la buena disponibilidad y voluntad de acceso de las organizaciones, aunque no todas las previamente seleccionadas quedaron de manera definitiva, ya que en determinadas ocasiones la presencia de la investigadora podía interpretarse como "fiscalizadora" del trabajo que ellas desempeñaban. Una vez incorporada a las dinámicas de las organizaciones, gracias al apoyo y a la buena comunicación con las educadoras y educadores, estas facilitaron datos sobre las niñas y las familias fruto de su trabajo desde hace años con las 


\section{Papeles de Trabajo No 33 - Julio 2017 - ISSN 1852-4508 \\ Centro de Estudios Interdisciplinarios en Etnolingüística y Antropología Socio-Cultural}

comunidades, y sin su ayuda no hubiera sido posible establecer el contacto y acceso a la población. En el caso del basurero de Chimalhuacán, el acceso a las familias y niñas trabajadoras, sin embargo, fue posible gracias a la labor de una comunidad religiosa.

Ahora bien ¿quiénes serían esos niños y niñas trabajadoras que observaríamos? ¿Cómo proceder para delimitar la noción de trabajador/a infantil? Conforme las especificidades de cada contexto y sensibles a ello elaboramos definiciones distintas. La delimitación de las unidades de análisis no ha sido una tarea sencilla, y desde un comienzo ha sido objeto de numerosos interrogantes.

En la investigación Argentina el trabajo infantil se recortó a todas aquellas actividades económicas realizadas por niños y niñas menores de 14 años (límite legalmente inferior para la admisión a un empleo $)^{8}$ y mayores de 5 , fueran o no remuneradas. Dichas actividades abarcaban tanto la producción de bienes y servicios como el trabajo doméstico excluyente y el limosneo. La OIT (2010) entiende que una actividad doméstica es excluyente en caso de que a) un niño/a deba dedicar todo su tiempo a dichas tareas viéndose privado de la posibilidad de ir a la escuela, situación en la que se considera a estas actividades como equivalentes a una actividad económica o b) cuando las tareas productivas familiares no pueden ser distinguidas de aquellas relativas a la reproducción familiar, porque el lugar en que se realizan aquellas y el lugar de habitación coinciden, o porque los ámbitos de producción y reproducción social están involucrados. La definición escogida difiere de otras, tanto en lo que respecta a las actividades que incluye y considera como laborales como así también con respecto al período de referencia tomado. Por ejemplo, en las investigaciones de la OIT el período de referencia que suele tomarse es el de una semana; en otros relevamientos de un mes y en otros un año. Dado el carácter estacional e intermitente que este tipo de actividad presenta, preferimos optar por el período más amplio, que es el de un año; asimismo la definición contemplaba una gran variedad de actividades que permitieran captar los distintos tipos de trabajo.

Una vez establecido este recorte en la definición, decidimos abordar a la totalidad de niños y niñas trabajadores del barrio escogido y a distintos miembros de la unidad doméstica que la integraban. En la investigación de México, para la selección de las "niñas" trabajadoras, el foco del estudio se concentró en aquellas con edades comprendidas entre los 4 y los 15 años, que trabajan en la calle y en espacios públicos (solas o acompañadas) dentro del ámbito urbano (específicamente en la Zona Metropolitana de Ciudad de México) y que de alguna manera conservan vínculos familiares. En cuanto a la distinción entre niñas y adolescentes, a pesar de que las fronteras están difuminadas por trayectorias vitales y laborales individuales, y es necesario considerarlas en cada caso particular, en el estudio de niñas se tuvieron en cuenta dos hechos significativos en la sociedad mexicana: la celebración de los 15 años que supone el rito de paso entre la infancia y la adolescencia, desde un punto de vista sociocultural y la consideración legal del trabajo a partir de los 16 años ${ }^{9}$, desde un punto de vista jurídico, quedando fuera de esta investigación el grupo de adolescentes de 16 a 18 años. Los criterios de selección de las unidades domésticas a partir de las niñas como punto de referencia se fueron determinando en función de algunas variables como el tipo de trabajo desempeñado por la niña, el tipo de unidad doméstica a la que pertenecía (teniendo en cuenta los criterios de residencia y parentesco), el lugar de trabajo y la edad.

Los modos en que procedimos a la definición de los ámbitos a observar y de los modos de plantear los recortes definicionales de la noción de trabajo infantil no hacen más que advertir sobre dos cuestiones. Una, la necesidad de seguir a esos niños y niñas por los distintos ámbitos en los que circulan, ya que creemos que para comprender mejor los procesos en que se ven implicados no basta con recortar un único espacio. Otra, la necesidad de no partir de definiciones unívocas sobre

8 En Argentina la modificación de la edad mínima de admisión del empleo fue progresiva: a raíz del cambio normativo que se generó en 2008 cuando se promulgó la ley número 26.390, la cual estableció que hasta mayo de 2010 la edad mínima fuera de 15 años, y luego quedara establecida en los 16 años. Cuando se dio dicho cambio ya habíamos comenzado nuestro trabajo de campo, por lo cual preferimos quedarnos con el límite original de los 14 años.

${ }^{9}$ Según la legislación mexicana, está prohibido el trabajo para menores de 16 años, aunque se permite para las y los menores de 14 y 15 en determinadas circunstancias. Los 16 años, por tanto, es la edad en la que se puede trabajar sin restricciones o condiciones especiales (Ley Federal del Trabajo. México, 1970). 
trabajo infantil, sino de construir tales definiciones atendiendo a las especificidades y características de cada contexto: legales, culturales, sociales, etc.

\section{Conclusiones}

En la medida en que reconocemos que nuestra posición como investigadoras es parte activa de la puesta en sentido de la realidad estudiada, en este caso, la realización de actividades laborales durante la infancia, recuperar, a través de una operación reflexiva el proceso de construcción del objeto de conocimiento y las definiciones metodológicas centrales para su estudio, contribuyó a delinear algunos de los elementos que componen un enfoque para estudiar el fenómeno de los/as niños/as y trabajadores/as.

Partimos de dos contextos distintos, con sus especificidades y de acuerdo a ello y a la sensibilidad producto de la particular mirada sobre cada uno de los mismos, fuimos construyendo el objeto de investigación y adoptando definiciones metodológicas y técnico- operativas ajustadas a su realidad. Como señalamos oportunamente, sin conocernos previamente, fuimos diagramando un recorrido con muchos puntos en común para estudiar el trabajo infantil urbano que aquí quisimos socializar. A saber:

-Entendemos que para estudiar a la niñez trabajadora es conveniente partir del enfoque propuesto por los childhood studies, con el cual compartimos plenamente la visión del niño/a, la niñez y los modos de aproximarse metodológicamente a su estudio. Reconocer las dinámicas y características del trabajo infantil requiere de aproximaciones que incorporen la perspectiva de los niños y niñas. -Creemos que para vislumbrar y acceder al fenómeno del trabajo infantil se requiere de observaciones en los múltiples espacios por los que circulan esos niños y niñas: no alcanza únicamente con observarlos trabajando o en sus barrios/hogares. Un análisis completo requiere de observaciones articuladas y no segmentadas.

- Apostamos a la construcción de un enfoque más integral que no considere al niño o niña en soledad, sino en su trama de relaciones: con personas adultas y con otros niños y niñas.

\section{Bibliografía}

- Alarcón, W. (1994): Ser niño. Una nueva mirada de la infancia en el Perú. UNICEF-IEPLima.

- Bar-Din, A. (comp.) (1995): Los niños marginados en América Latina. Una antología de estudios psicosociales -Centro de Investigaciones Interdisciplinarias en Humanidades. Universidad Nacional Autónoma de México- México.

- Boyden, J- y Ennew, J. (1997): Children in focus: A manual for participatory research with children-Rädda Barnen- Estocolmo.

- Casas, F. (1998): Infancia: perspectivas psicosociales- Paidós Ibérica-Barcelona.

- Castillo, M. (2004): "El protagonismo infantil o las posibilidades y límites del construccionismo" en Infancia y Adolescencia en América Latina. Aportes desde la Sociología -VV.AA.- IFEJANT-Lima.

- Christensen, P. (2004): "Children's participation in ethnographic research: issues of power and representation" en Children \& Society, 18 (2)- NCB- (165-176).

- Cohn, C. (2007): "O desenho das crianças e o antropólogo: reflexões a partir das crianças mebengokré-xikrin" VI Reunión de Antropología del Mercosur. Montevideo: Uruguay.

- Eder, D. y Corsaro, W. (1999): "Ethnographic studies of children and youth. Journal of contemporary ethnography" 28 (5)- Sage- (20-531).

- Gaitán, L. (2006): Sociología de la Infancia -Síntesis- Madrid.

- Gaitán, L. y Liebel, M. (2011): Ciudadanía y derechos de participación de los niños. SíntesisMadrid.

- Grover, S. (2004): "Why Won't They Listen to Us? On Giving Power and Voice to Children Participating in Social Research" en Childhood, 11 (1) - Sage- (81-93). 
- Guber, R. (1991): El salvaje metropolitano. Reconstrucción del conocimiento social en el trabajo de campo -Paidós- Buenos Aires.

- Hidalgo, C. (2006): "Reflexividades" en Cuadernos de Antropología Social 23- Facultad de Filosofía y Letras de la UBA- (45-56).

- Lahire, B. (2007): "Infancia y adolescencia: de los tiempos de socialización sometidos a constricciones múltiples" en Revista de Antropología Social 16- Universidad Complutense de Madrid- (21-38).

- OIT (2010): Panorama Laboral 2010- OIT-Lima.

- Pollock, L. (1983): Los niños olvidados. Relaciones entre padres e hijos de 1500 a 1900Fondo de Cultura Económica-México.

- Rausky, M. E. (2010): "Los abordajes metodológicos en el campo de estudios sociales sobre la niñez: principales tendencias y perspectivas" en Renglones, Revista arbitrada en ciencias sociales y humanidades-62- Iteso- (134-158).

- Rodríguez, I. (2005): "Revisando críticamente el discurso sobre el impacto de la sociedad de la información en la población infantil: el problema del aislamiento social" en Papers-77UAB- (157-178).

- Sirota, R. (2001): "Emergencia de una sociología da infancia: evolucao du objeto e olhar" en Cadernos de pesquisa 112- Editora Autores Asociados-(7-31)

- Szulc, A. (2004): "La antropología frente a los niños: de la omisión a las culturas infantiles". Ponencia presentada en el VII Congreso Argentino de Antropología. Realizado en Córdoba, el 25 al 28 de mayo de 2004. 